\title{
Multipole polarizability of a nanodimer in optical waves
}

P. Grahn

patrick.grahn@aalto.fi

\section{A. Shevchenko}

\section{Kaivola}

Department of Applied Physics, Aalto University, P.O. Box 13500, FI-00076 Aalto, Finland

Department of Applied Physics, Aalto University, P.O. Box 13500, FI-00076 Aalto, Finland

In this work we study the interaction of visible light with plasmonic nanodimers that, under particular illumination conditions, do not exhibit any electric dipole excitations. It has previously been found out that the dipole suppression phenomenon disappears when the illumination direction is reversed. As a consequence, a homogeneous nanomaterial consisting of such nanodimers can be expected to be spatially dispersive, such that the conventional electric polarization vanishes for certain directions of light propagation. In order to reveal the complete picture of the light-nanodimer interaction, we analyze the multipole excitations in a dimer at various illumination angles. In particular, we introduce an analytical model for the multipole polarizability tensors of the dimer that, in contrast to conventional polarizability tensors, depend on the light propagation direction. The model is verified by rigorous numerical calculations. It can be used to gain insight into the properties of optical nanomaterials, such as metamaterials, in which higher-order multipoles can be efficiently excited. [DOI: http://dx.doi.org/10.2971/jeos.2013.13009]

Keywords: Optical multipoles, nanoscatterers, multipole polarizability, nanomaterials, spatial dispersion

\section{INTRODUCTION}

The interaction of light with subwavelength-size particles is usually accurately described in terms of electric dipole excitations. Consequently, the widely used electrodynamics of continuous media rely upon the dipole approximation of the material constituents. As an exception, in custom-designed optical nanomaterials the unit cell response can be tuned in such a way that other multipoles are excited as well [1], which leads to various interesting optical phenomena [2] and extraordinary optical effects [3]. In order for the extraordinary phenomena to be strong, the light scattering by higher-order multipoles must be significant [4]. Furthermore, in order to treat the nanomaterial as a homogeneous one, the dimensions of the unit cell must be much smaller than the illumination wavelength $[5,6]$. Thus, we seek to design a sub-wavelength unit cell that will provide a considerable polarizability for the higher-order multipoles.

For visible light, it has been shown that a pair of silver bars can exhibit a resonance with pronounced electric quadrupole and magnetic dipole excitations [7]. More recently, it was shown that a nanodimer composed of two silver discs can be tuned to completely suppress the electric dipole excitation by light at normal incidence [4]. This opens up a possibility to create a homogeneous nanomaterial in which the light-matter interaction is completely governed by the higher-order multipoles for a given direction of light propagation. The dominance of the higher-order multipoles can also be used to enhance electric dipole-forbidden transitions in quantum mechanical systems [8]. So far, the optical properties of such electric dipole- free nanodimers, including their response at various incidence angles, have not been thoroughly investigated.

In this paper, we present a complete description of the interaction of optical plane waves with a nanodimer structure that exhibits electric dipole suppression. The interaction is characterized in terms of optical multipole polarizabilities of the nanodimer. Analytical expressions for these polarizabilities at arbitrary incidence angles are derived and verified numerically. This characterization is especially valuable for obtaining spatially dispersive optical parameters [9] that describe nanomaterials consisting of such dimers. In Section 2, we demonstrate the electric dipole suppression in a disc nanodimer and show the dependence of the multipole excitations on the orientation of the dimer with respect to the incident light. The dipole suppression is shown to occur for a wide range of incidence angles. In Section 3, we introduce multipole polarizability tensors that fully characterize the angle-dependent optical response of the dimer through a few simple analytical expressions. Finally, in Section 4 we summarize our results.

\section{ELECTRIC DIPOLE SUPPRESSION IN DISC NANODIMERS}

We consider a disc nanodimer that consists of two axisaligned silver discs arranged as in Figure 1(a). The dimensions of the nanodimer are chosen to be $R_{1}=15 \mathrm{~nm}, R_{2}=20 \mathrm{~nm}$ and $h_{1}=h_{2}=s=10 \mathrm{~nm}$. The size of the dimer is there- 

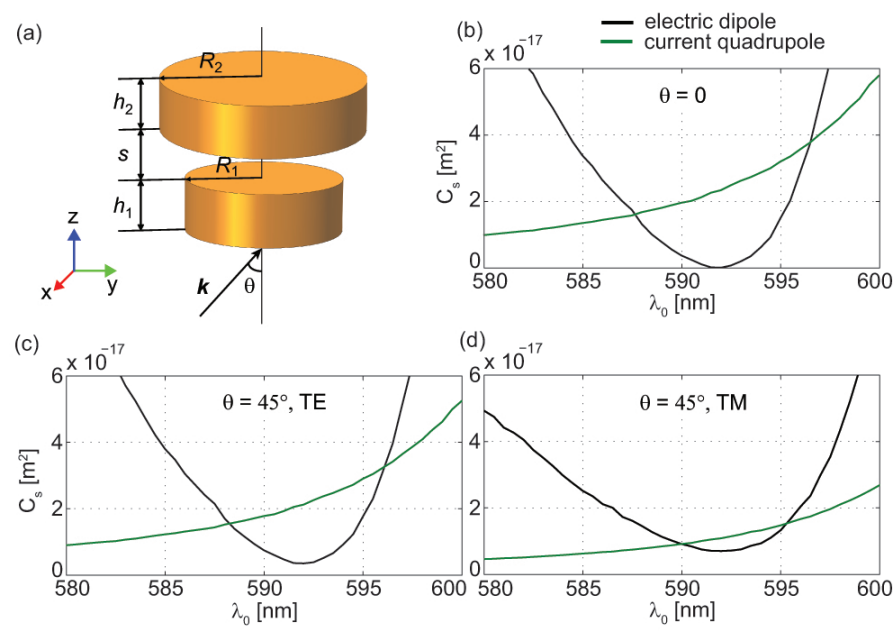

FIG. 1 (a) Geometry of the disc nanodimer. (b-d) Spectra of the multipole contributions to the scattering cross section of the nanodimer. The incident light has (b) $\theta=0$, (c) $\theta=45^{\circ}$ and TE polarization, or (d) $\theta=45^{\circ}$ and TM polarization.

fore much smaller than the optical wavelength. The structure is considered to be illuminated with an optical plane wave that propagates at an angle $\theta$ with respect to the dimer axis. For each illumination angle $\theta$, the incident light can be decomposed into two orthogonal polarization components. We shall call the component with the electric (magnetic) field perpendicular to the dimer axis as the TE (TM) component.

We start by numerically calculating the electromagnetic field scattered by the nanodimer using the computer software COMSOL Multiphysics. The relative electric permittivity of silver is obtained from [10]. For illumination at normal incidence from the side of the smaller disc $(\theta=0)$, the electric currents in the two discs can oscillate out of phase with respect to each other, such that the net electric dipole moment $\mathbf{p}$ is canceled. For our nanodimer, this dipole suppression occurs at a vacuum wavelength of $\lambda_{0}=592 \mathrm{~nm}$ when the surrounding medium is a dielectric of refractive index 1.5 (see Figure 1(b)). At this wavelength, the multipole decomposition [11] of the scattered field reveals that the nanodimer is equivalent to a polarizable point current quadrupole. This current quadrupole, being characterized by two opposite timeharmonic currents, is a superposition of the classical magnetic dipole and electric quadrupole. The wavelength at which $\mathbf{p}$ disappears can be tuned, e.g., by changing the disc separation $s[4]$.

Next, we consider the case of oblique incidence that has not been considered previously. The multipole scattering cross sections for TE and TM polarized illuminations at $\theta=45^{\circ}$ are depicted in Figures 1(c) and 1(d), respectively. For the TE polarized illumination, the electric dipole moment is still well suppressed at $\theta=45^{\circ}$. The situation is different for the TM polarized light, since the polarization component parallel to the dimer axis provides an additional contribution to the electric dipole scattering. Thus, at $\theta=45^{\circ}$ the scattering contributions of the electric dipole and the current quadrupole are comparable at $\lambda_{0}=592 \mathrm{~nm}$. For both polarizations, the spectral location of the minimum of the electric dipole moment is seen to be independent of $\theta$.

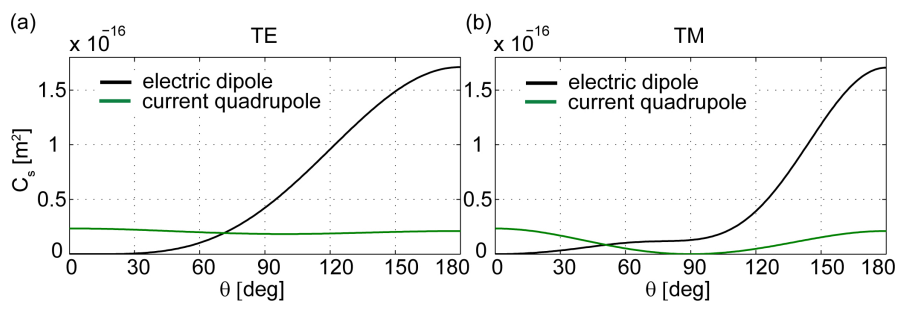

FIG. 2 Comparison of the multipole contributions to the nanodimer scattering cross section as a function of the angle of incidence for (a) TE and (b) TM polarized light.

Since the wavelength at which the electric dipole gets suppressed is independent of the angle of incidence, it is meaningful to analyze the scattering cross sections at this particular suppression wavelength. In Figure 2 the scattering cross sections are depicted versus the angle of incidence for the two orthogonal polarizations. The current quadrupole is found to be the dominating multipole up to the incidence angle of $\theta=60^{\circ}$ for the TE polarization and $\theta=45^{\circ}$ for the TM polarization. Therefore, in a nanomaterial composed of such nanodimers, the electric dipole suppression can be expected to take place for a wide range of light propagation directions. Furthermore, on the basis of the Fourier plane wave decomposition, it is evident that the dipole suppression can also be achieved when using tightly focused optical beams or beams with large wavefront curvatures.

From Figure 2 we notice that the excited dipole moment increases rapidly as $\theta$ is increased above $90^{\circ}$. For the TE polarization, the strength of the excited current quadrupole changes only weakly with $\theta$. For the TM polarization, on the other hand, the current quadrupole is suppressed at $\theta=90^{\circ}$. This can be explained by the fact that the $\mathrm{z}$-component of the electric field is unable to efficiently excite higher-order multipoles. In the next section we present a simple analytic model that successfully explains the features in Figure 2.

\section{MULTIPOLE POLARIZABILITY MODEL FOR NANODIMERS}

In order to gain physical insight into the dependence of the nanodimer multipole excitations on the incidence angle, we develop a simple analytical model for the multipole polarizability. In this model, we assume for simplicity that the dipole polarizability components of the individual nanodiscs of the dimer are independent of the illumination direction. Let us consider the example of a time-harmonic TE polarized illumination with the complex amplitude of the electric field given as $\mathbf{E}(\mathbf{r})=\hat{\mathbf{x}} E_{0} \exp [i k(z \cos \theta+y \sin \theta)]$, where $k$ is the wavenumber in the surrounding medium. In this case, the effective dipole moment of the disc pair, when considered as a point particle in the center of the dimer, can be written in the form

$$
\mathbf{p}(\theta)=\left[\alpha_{1} \exp \left(-i \frac{k d}{2} \cos \theta\right)+\alpha_{2} \exp \left(i \frac{k d}{2} \cos \theta\right)\right] \mathbf{E}_{0},
$$

where $\alpha_{j}$ is the effective polarizability of disc $j$ as a part of the nanodimer, $d$ is the center-to-center separation between the discs and $\mathbf{E}_{0}=\hat{\mathbf{x}} E_{0}$. Defining the quantities $\tilde{\alpha}_{1}=\alpha_{1} \exp (-i k d / 2)$ and $\tilde{\alpha}_{2}=\alpha_{2} \exp (i k d / 2)$, we rewrite 
Eq. (1) as

$$
\begin{aligned}
\mathbf{p}(\theta)=\{ & \tilde{\alpha}_{1} \exp \left[-i \frac{k d}{2}(\cos \theta-1)\right] \\
& \left.+\tilde{\alpha}_{2} \exp \left[i \frac{k d}{2}(\cos \theta-1)\right]\right\} \mathbf{E}_{0} .
\end{aligned}
$$

For a subwavelength-size nanodimer the phase delay between the discs is small, i.e., $k d<<\pi$. Writing the exponential functions in Eq. (2) as their Taylor series and neglecting all terms of higher order than $(k d)^{1}$, we obtain

$$
\mathbf{p}(\theta) \approx \mathbf{p}(0)+i k d \frac{1-\cos \theta}{2}\left(\tilde{\alpha}_{1}-\tilde{\alpha}_{2}\right) \mathbf{E}_{0} .
$$

In order for a nanodimer to exhibit electric dipole suppression at normal incidence, the dipole moments in the individual discs must be of equal amplitudes and oscillate out of phase such that $\tilde{\alpha}_{2}=-\tilde{\alpha}_{1}$, yielding $\mathbf{p}(0)=0$. Eq. (3) then suggests that if the illumination direction is reversed, an electric dipole moment of $\mathbf{p}=2 i k d \tilde{\alpha}_{1} \mathbf{E}_{0}$ is excited. If the incident light is TM polarized, in which case $\mathbf{E}(\mathbf{r})=(\hat{\mathbf{y}} \cos \theta-$ $\hat{\mathbf{z}} \sin \theta) E_{0} \exp [i k(z \cos \theta+y \sin \theta)]$, the disc symmetry allows us to use the same $\tilde{\alpha}_{1}$ and $\tilde{\alpha}_{2}$ to evaluate the $y$-component of p. However, the response to the z-component of the incident electric field is described by different polarizabilities of the discs. These polarizabilities are found to be non-resonant and have nearly equal arguments. Thus, the second term in Eq. (3) can be neglected for $p_{z}$, and the corresponding polarizability of the dimer becomes independent of $\theta$.

The dipolar response of the nanodimer to arbitrary planewave illumination can be characterized by introducing a polarizability dyadic $\overleftrightarrow{\alpha}$ that depends on $\theta$. The excited electric dipole moment is then expressed as

$$
\mathbf{p}(\theta)=\overleftrightarrow{\alpha}(\theta) \cdot \mathbf{E}_{0}
$$

Since the nanodimer possesses axial symmetry, we propose a diagonal dipole polarizability dyadic with the elements

$$
\begin{aligned}
\alpha_{x x} & =\alpha_{y y}=a_{1}+\frac{1-\cos \theta}{2} \tilde{a}_{1}, \\
\alpha_{z z} & =a_{2},
\end{aligned}
$$

where the coefficients $a_{1}, \tilde{a}_{1}$ and $a_{2}$ are independent of $\theta$. The form of Eq. (5) is chosen in accordance with Eq. (3) such that $a_{1}=0$ when the nanodimer is tuned to have electric dipole suppression for $\theta=0$, and the polarizability for the opposite illumination direction is $\tilde{a}_{1}$. The element $\alpha_{z z}$ is assumed to be constant as explained above. According to Eqs. (5) and (6), calculating or measuring the dipole response at $\theta=0, \theta=\pi / 2$ and $\theta=\pi$ is enough to determine the dipole response at any incidence angle.

The excitation of the current quadrupoles is described by the current quadrupole polarizability triadic $\overleftrightarrow{\beta}$ as

$$
\overleftrightarrow{\mathbf{Q}}(\theta)=\stackrel{\leftrightarrow}{\beta}(\theta) \cdot \mathbf{E}_{0} .
$$

Under the dipole suppression conditions, the main excitations in the nanodimer are the current quadrupoles $Q_{x z}$ and $Q_{y z}$ (see [4]). However, also minor contributions from the current quadrupoles $Q_{z x}$ and $Q_{z y}$ are present. All other current

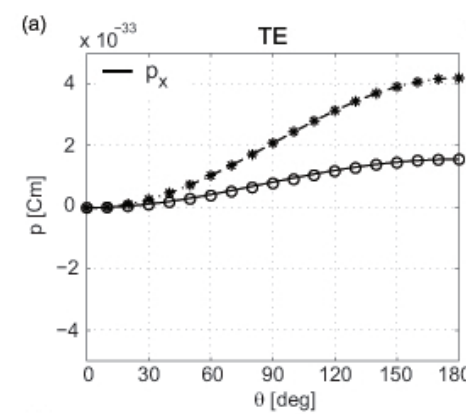

(b) $\times 10^{-33} \quad \mathrm{TM}$

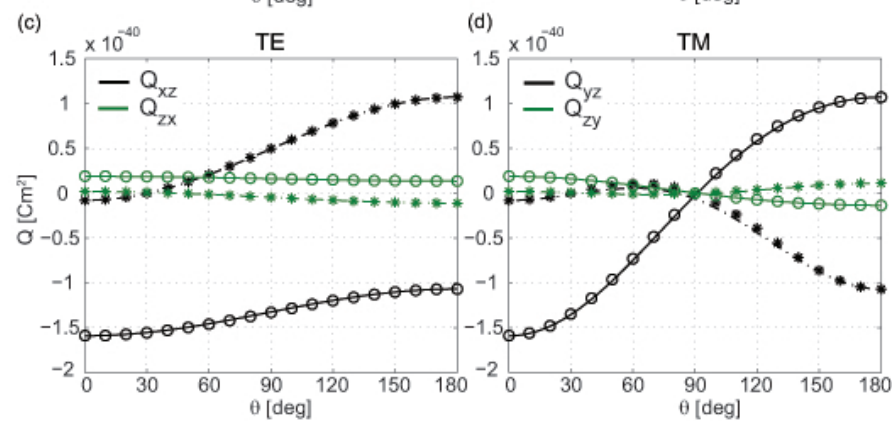

FIG. 3 Components of the current multipole moments excited in the silver disc nanodimer by a plane wave with amplitude $1 \mathrm{~V} / \mathrm{m}$ and vacuum wavelength $\lambda_{0}=592 \mathrm{~nm}$. The moments $p_{x}, Q_{x z}$ and $Q_{z x}$ are excited by a TE polarized wave, while the moments $p_{y}, p_{z}, Q_{y z}$ and $Q_{z y}$ are excited by a TM wave. The real parts (solid lines) and imaginary parts (dashed lines) of the numerically computed moments are shown along with the real parts (circles) and imaginary parts (stars) of the moments described by the analytical Eqs. (4)-(9).

quadrupoles are found to be negligible. Analogously to the dipoles, we propose a quadrupole polarizability triadic with the following non-zero elements

$$
\begin{aligned}
& \beta_{x z x}=\beta_{y z y}=b_{1}+\frac{1-\cos \theta}{2} \tilde{b}_{1} \\
& \beta_{z x x}=\beta_{z y y}=b_{2}+\frac{1-\cos \theta}{2} \tilde{b}_{2}
\end{aligned}
$$

where the nanodimer geometry and the interaction symmetry are taken into account. The optical response of the disc nanodimer is therefore considered to be completely described by the four different multipole polarizabilities $\alpha_{x x}, \alpha_{z z}, \beta_{x z x}$, and $\beta_{z x x}$.

In order to verify the proposed analytical expressions for the nanodimer polarizabilities, we numerically calculate the excited multipoles as functions of $\theta$. The calculated components of the excited electric dipole moment $p_{x}$ for the TE polarization and $p_{y}$ and $p_{z}$ for the TM polarization are shown in Figures 3(a) and 3(b), respectively, by the solid and dashed curves standing for the real and imaginary parts of the moments. In the same figure (see circles and stars) we show the results obtained from Eqs. (4)-(6) with $a_{1}=0, \tilde{a}_{1}=(4.2+1.6 i) \times 10^{-33} \mathrm{Cm}^{2} / \mathrm{V}$ and $a_{2}=1.2 \times 10^{-33} \mathrm{Cm}^{2} / \mathrm{V}$. These coefficients were calculated by using the numerical data obtained for $\theta=0, \theta=\pi / 2$ and $\theta=\pi$.

Similarly, the excited current quadrupole moments $Q_{x z}$ and $Q_{z x}$ for the TE polarization and $Q_{y z}$ and $Q_{z y}$ for the TM polarization are shown in Figures 3(c) and 3(d), respectively. The real and imaginary parts of the moments are shown by the solid and dashed lines, respectively. For the current 
quadrupole polarizability coefficients we have

$$
\begin{aligned}
& b_{1}=(-1.59-0.08 i) \times 10^{-40} \mathrm{Cm}^{3} / \mathrm{V}, \\
& \tilde{b}_{1}=(0.52+1.15 i) \times 10^{-40} \mathrm{Cm}^{3} / \mathrm{V}, \\
& b_{2}=(0.19+0.02 i) \times 10^{-40} \mathrm{Cm}^{3} / \mathrm{V} \text { and } \\
& \tilde{b}_{2}=(-0.05-0.13 i) \times 10^{-40} \mathrm{Cm}^{3} / \mathrm{V} .
\end{aligned}
$$

Figure 3 shows a remarkable agreement between the analytical model introduced by Eqs. (4)-(9) and the rigorous numerical calculations. At angles $\theta \approx \pi / 2$, there is a minor deviation in the analytical multipole moments $p_{y}, Q_{y z}$ and $Q_{z y}$ for the TM polarized light. This deviation originates from the fact that also the z-component of the electric field is able to partially excite these multipoles. This coupling can be added to the model by introducing additional non-zero polarizability elements, which can be solved from the multipole response at $\theta=\pi / 2$. The magnitudes of these additional elements are, however, quite small and therefore they do not considerably affect the total light-nanodimer interaction.

The multipole polarizability model introduced here is based on considering the interference of the waves scattered by the two spatially separated dipolar particles. Thus, the model is not restricted to the case of the disc nanodimer and the electric dipole suppression phenomenon, but can be applied for a general description of light interaction with pairs of subwavelength particles.

The angular dependence of the multipole excitations is responsible for the spatial dispersion in optical nanomaterials. It is therefore important to have a complete picture of this dependence for the nanoscatterers composing such nanomaterials. If, for example, the nanomaterial consists of nanoscatterers of the type considered here, one can obtain the macroscopic dipole and quadrupole polarizations for each propagation direction and polarization of the field by multiplying the calculated $\mathbf{p}$ and $\stackrel{\leftrightarrow}{\mathbf{Q}}$ with the density of the particles in the material. The multipole polarizabilities in this case have to be calculated by taking into account the interaction between the particles in the nanomaterial.

\section{CONCLUSIONS}

We have shown that subwavelength-size nanodimers can exhibit electric dipole suppression for a wide range of incidence angles. In these cases, the light-nanodimer interaction can be described solely by the current quadrupole excitations. For a simplified and more insightful description of this interaction at arbitrary angles of incidence, we have presented an analytical model that introduces dipole and quadrupole polarizability tensors for the nanodimers. This simple model shows an excellent agreement with rigorous numerical calculations. Knowing the analytical expressions for the plane wave response of the dimer at all possible incidence angles, the response to an arbitrary propagating field is easily obtained by superposition. The introduced multipole polarizabilities can also be used to determine the spatially dispersive macroscopic optical parameters of a homogenized optical nanomaterial constructed of resonant nanodimers.

\section{ACKNOWLEDGEMENTS}

This work was funded by the Academy of Finland (project no. 134029).

\section{References}

[1] C. Rockstuhl, C. Menzel, S. Mühlig, J. Petschulat, C. Helgert, C. Etrich, A. Chipouline, et al., "Scattering properties of meta-atoms," Phys. Rev. B 83, 245119 (2011).

[2] R. E. Raab, and 0. L. de Lange, Multipole Theory in Electromagnetism (Oxford, New York, 2005).

[3] W. Cai, and V. Shalaev, Optical Metamaterials: Fundamentals and Applications (Springer, New York, 2009).

[4] P. Grahn, A. Shevchenko, and M. Kaivola, "Electric dipole-free interaction of visible light with pairs of subwavelength-size silver particles," Phys. Rev. B 86, 035419 (2012).

[5] C. R. Simovski, "On electromagnetic characterization and homogenization of nanostructured metamaterials," J. Opt. 13, 013001 (2011).

[6] N. A. Mortensen, M. Yan, 0. Sigmund, 0. Breinbjerg, "On the unambiguous determination of effective optical properties of periodic metamaterials: a one-dimensional case study," J. Europ. 0pt. Soc. Rap. Public. 5, 10010 (2010).

[7] D. J. Cho, F. Wang, X. Zhang, and Y. R. Shen, "Contribution of the electric quadrupole resonance in optical metamaterials," Phys. Rev. B 78, 121101 (2008).

[8] R. Filter, S. Mühlig, T. Eichelkraut, C. Rockstuhl, and F. Lederer, "Controlling the dynamics of quantum mechanical systems sustaining dipole-forbidden transitions via optical nanoantennas," Phys. Rev. B 86, 035404 (2012).

[9] B. Compf, B. Krausz, B. Frank, and M. Dressel, “k-dependent optics of nanostructures: Spatial dispersion of metallic nanorings and split-ring resonators," Phys. Rev. B 86, 075462 (2012).

[10] P. B. Johnson and R. W. Christy, "Optical constants of the noble metals," Phys. Rev. B 6, 4370-4379 (1972).

[11] P. Grahn, A. Shevchenko, and M. Kaivola, "Electromagnetic multipole theory for optical nanomaterials," New J. Phys. 14, 093033 (2012). 